\title{
Commentary
}

\section{Impact of COVID-19: Perspectives from Sport and Exercise Medicine}

Dinesh Sirisena, ${ }^{1,3}$ FFSEM (UK\&I), FFSEM (Ire), FAMS (Sports Med), Mandy Zhang, ${ }^{2,3}$ MbBChBAO (Ire), MSpMed (Aust), FAMS (Sports Med),

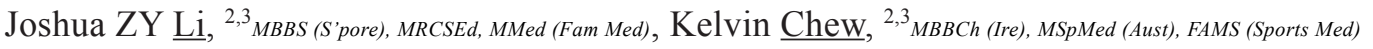

\section{COVID-19 and the Community}

The coronavirus disease 2019 (COVID-19) pandemic is a global crisis that has resulted in much of the world being placed on lockdown to limit the spread of the virus. Here in Singapore, the lockdown was imposed on 7 April 2020. The cessation of organised sporting activities and much of sport itself, has led to limited exercise opportunities and this is concerning for the medical fraternity. The 2010 World Health Organisation document on physical activity (PA) ${ }^{1}$ highlighted the importance of exercise for health and its role in the prevention and management of chronic disease. The lockdown potentially sets back progress made by the local population and may trigger unhealthy eating habits, such as irregular meal times, snacking, increased alcohol consumption and stress eating. ${ }^{2}$ Sport and Exercise Medicine (SEM) practitioners must be ready to support the community and athletes once restrictions are lifted.

Reports suggest that COVID-19 is more severe in individuals with diabetes, ${ }^{3}$ hypertension ${ }^{4}$ and obesity. ${ }^{5}$ In addition to causing a cytokine storm from overproduction of proinflammatory markers, leading to exuberant systemic inflammation and multi-organ failure, ${ }^{6}$ the causative SARS-CoV-2 virus causes cardiovascular complications such as cardiomyopathy, myocarditis and malignant ventricular arrhythmia. Indeed, $19.7 \%$ of hospitalised COVID-19 patients had myocardial injury as a complication. ${ }^{7}$ Thus, it is imperative that despite the lockdown, patients with chronic medical conditions maintain PA and optimise disease control.

Burnout is common among healthcare professionals, and this has been exacerbated by COVID-19. ${ }^{8,9}$ Amongst the wider population, restrictions to normal life have triggered psychological symptoms, such as depression and anxiety. Stressors during lockdown include the quarantine duration, infection fears, frustration, boredom, inadequate supplies, inadequate information, financial loss and stigma. ${ }^{10}$ Exercise is known to help with mild to moderate mental health conditions ${ }^{11}$ and should be utilised to cope with stress during this period.

School closures and the impact on child health is another concern. ${ }^{12}$ With prolonged home-based learning, restricted outdoor playtimes, and increased screen time against a backdrop of rising childhood obesity, healthcare systems must prepare for the potential consequences of this pandemic.

Equally at risk are the elderly, who are more susceptible to severe COVID-19 infections. They must physically distance themselves from others; however, being at higher risk for chronic disease, depression and low cardiorespiratory fitness, they have an imperative need to maintain PA to counter the health consequences of a lockdown.

As SEM practitioners, we must advocate safe PA. While some people refrain from exercising, those who were previously sedentary may utilise PA as a way of coping with the current restrictions. Unfortunately, for individuals who were once physically inactive, there may be a lack of knowledge of fitness levels, safe progression to exercises, injury prevention and equipment use. Thus, education must continue through multimedia communication like online webinars, posts on social media and healthcare websites. We should be ready to answer queries on exercise modalities, online programmes, progress monitoring and injury management.

\footnotetext{
${ }^{1}$ Sports Medicine Centre, Khoo Teck Puat Hospital, Singapore

${ }^{2}$ Department of Sport \& Exercise Medicine, Changi General Hospital, Singapore

${ }^{3}$ Sports Medicine Association Singapore, Singapore

Address for Correspondence: Dr Dinesh Sirisena, Sports Medicine Centre, Khoo Teck Puat Hospital, 90 Yishun Central, Singapore 768828.

Email: dinesh.sirisena@ktph.com.sg
} 


\section{COVID-19 and Athletes}

The COVID-19 pandemic also poses challenges for competitive athletes who aim to peak at certain races, or within a specific period, through the intricate science of periodisation to optimise performance. ${ }^{13}$ The disruption of training programs and closure of sports and recreational facilities will inevitably lead to a degree of physical deconditioning, but psychologically, there could have been grief, stress and frustration, compounded by the removal of social support networks and training routines. ${ }^{14}$ Significantly, the postponement of major events such as the 2020 Tokyo Olympic Games have undoubtedly raised anxieties and uncertainties amongst athletes, who must now devote additional time and reprioritise other life events to be at their peak. Thus, sports psychologists and SEM practitioners should be cognizant of this and work together to maintain the physical and mental wellbeing of athletes.

Exercise and immunity have a proven relationship; the 'J-shaped hypothesis' describes the association between PA and respiratory tract infection. ${ }^{15}$ Prolonged high-intensity training has been associated with decreased $\mathrm{T}$ and natural killer cell function, causing transient immune perturbations lasting from $3-72 \mathrm{hrs} .{ }^{16}$ Therefore, during the COVID-19 pandemic, athletes are generally advised to limit training sessions to $<60$ minutes and $<80 \%$ of maximal ability. For elite athletes accustomed to high-intensity training, evidence suggests this may continue during the pandemic, provided there is no sudden increase in training load. ${ }^{17}$

Pre-participation screening for elite athletes is an important aspect of SEM practice; this will be particularly important amongst those who suffered from, or were at a high risk of COVID-19 infection. Health providers should be mindful of potential cardiovascular sequelae; in addition to a detailed history and physical examination, physicians may utilise electrocardiograms, echocardiograms, exercise tolerance tests to decide suitability to return to play, and may consider COVID-19 antibody testing, depending on reliability, to identify confirmed cases. ${ }^{18,19}$ It may also be necessary to consult a wider team (cardiologists, medical support staff, coaches and parents) to identify affected individuals and try to safeguard athletes.

Indeed, the ideal time of return to sport for elite athletes following COVID-19 infections has yet to be determined. Traditionally, the 'neck check rule' applies, where athletes can attempt to resume training if symptoms were limited above the neck. More generalised symptoms below the neck, such as fever and fatigue, require full resolution. ${ }^{20}$ However, with COVID-19 being a relatively new infection, associated with multi-organ complications, ${ }^{7}$ there remain many uncertainties. Most athletes may resume training once symptoms have resolved for 2 weeks to limit cardiorespiratory symptoms, although a more conservative, graduated approach with prolonged rest and clearance should be considered.

Ultimately, the COVID-19 pandemic will inevitably impact athletes in different ways; SEM providers will need to validate and support them through their concerns with a multi-faceted approach.

\section{Conclusion}

Since the commencement of this article, the lockdown was extended until 1 June 2020. This will undoubtedly further challenge the population, in terms of maintaining health and mental well-being. The importance of PA and exercise will be further highlighted, particularly when 'lockdown fatigue' sets in. It is crucial that SEM practitioners reflect on our practice, seek ways to evolve with the times and keep up to date with national and international guidelines. Amid uncertainties, the only constant is that the world post-COVID-19 will be significantly different. In addition to being prepared to adapt in whatever way is necessary, it is also important that we ask questions of ourselves and identify where knowledge gaps might exist (Figure). By doing so, we will hopefully be ready to support our patients and the nation during this time of need. 


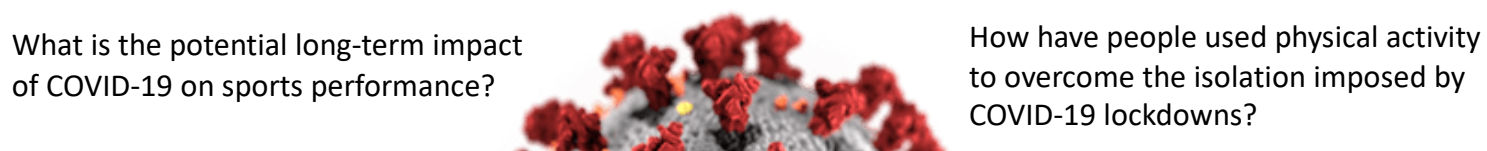

How will physical distancing measures impact the way recreational, competitive and elite athletes train?

How will COVID-19 change massive sports participation and spectatorship? COVID-19 lockdowns?

Has there been increased public awareness of the importance of physical activity in health and wellness?

Will COVID-19 be a barrier to physical activity as a treatment for chronic medical and mental health conditions?

Figure. Future Considerations of COVID-19 in Sport

\section{REFERENCES}

1. World Health Organisation. Global recommendations on physical activity for health. Geneva: World Health Organisation; 2010; 1-58.

2. Naja F, Hamadeh R. Nutrition amid the COVID-19 pandemic: a multi-level framework for action. Eur J Clin Nutr 2020; 74:1117-21.

3. Huang I, Lim MA, Pranata R. Diabetes mellitus is associated with increased mortality and severity of disease in COVID-19 pneumonia - A systematic review, meta-analysis, and meta-regression. Diabetes Metab Syndr 2020;14:395-403.

4. Cook TM. The importance of hypertension as a risk factor for severe illness and mortality in COVID-19. Anaesthesia 2020;75:976-77.

5. Finer N, Garnett SP, Bruun JM. COVID-19 and obesity. Clinical Obes 2020;10:e12365.

6. Bhandari S, Sharma S, Bhargava A, Keswani P, Sharma R, Shekhawat A. Inflammatory markers in COVID-19. Ann Acad Med Singap 2020;49:393-7.

7. Shi S, Qin M, Shen B, Cai Y, Liu T, Yang F, et al. Association of cardiac injury with mortality in hospitalized patients with COVID-19 in Wuhan, China. JAMA Cardiol 2020;5:802-10.

8. Ho CS, Chee CY, Ho RC. Mental health strategies to combat the psychological impact of COVID-19 beyond paranoia and panic. Ann Acad Med Singap 2020;49:155-60.

9. Goh SSN, Chia MYC. Anxiety and morale in front-line healthcare workers during the coronavirus disease 2019 (COVID-19) outbreak at the national screening centre in Singapore. Ann Acad Med Singap 2020;49:259-62.

10. Brooks SK, Webster RK, Smith LE, Woodland L, Wessely S, Greenberg $\mathrm{N}$, et al. The psychological impact of quarantine and how to reduce it: rapid review of the evidence. Lancet 2020;395:912-20.

11. Rosenbaum S, Tiedemann A, Sherrington C, Curtis J, Ward PB. Physical activity interventions for people with mental illness: A systematic review and meta-analysis. J Clin Psychiatry 2014;75:964-74.
12. Rundle AG, Park Y, Herbstman JB, Kinsey EW, Wang YC. COVID-19-related school closings and risk of weight gain among children. Obesity (Silver Spring) 2020;28:1008-9.

13. Lyakh V, Mikołajec K, Bujas P, Witkowski Z, Zając T, Litkowycz $\mathrm{R}$, et al. Periodization in team sport games - a review of current knowledge and modern trends in competitive sports. Journal Hum Kinet 2016;54:173-80.

14. Toresdahl BG, Asif IM. Coronavirus disease 2019 (COVID-19): considerations for the competitive athlete. Sports Health 2020;12:221-4.

15. Chamorro-Viña C, Fernandez-del-Valle M, Tacón AM. Excessive exercise and immunity: the J-shaped curve. In: Robert-McComb JJ, Norman RL, Zumwalt M, editors. The Active Female. New York: Springer; 2014;357-72.

16. Nieman DC. The immune response to prolonged cardiorespiratory exercise. Am J Sports Med 1996;24:S98-S103.

17. Malm C. Susceptibility to infections in elite athletes: the S-curve. Scand J Med Sci Sports 2006;16:4-6.

18. Baggish AL, Drezner JA, Kim JH, Martinez MW, Prutkin JM. The resurgence of sport in the wake of COVID-19: cardiac considerations in competitive athletes. British Journal of Sports Medicine Blog 2020. Available at: https://blogs.bmj.com/ bjsm/2020/04/24/the-resurgence-of-sport-in-the-wake-of-covid19-cardiac-considerations-in-competitive-athletes/. Accessed on 2nd May 2020.

19. Drezner JA, O'Connor FG, Harmon KG, Fields KB, Asplund CA, Asif IM, et al. AMSSM position statement on cardiovascular preparticipation screening in athletes: current evidence, knowledge gaps, recommendations and future directions. Br J Sports Med 2017;51:153-67.

20. Harris MD. Infectious disease in athletes. Curr Sports Med Rep $2011 ; 10 ; 84-9$. 